\title{
Efficient Quantization for Feedback Controlled Networks with Type II Hybrid ARQ
}

\author{
Marco Levorato*, Leonardo Badia ${ }^{\dagger}$ and Michele Zorzi* \\ * Dept. of Information Engineering, University of Padova, via Gradenigo 6/B, 35131 Padova, Italy. \\ $\dagger$ IMT Lucca Institute for Advanced Studies, piazza S. Ponziano 6, 55100 Lucca, Italy. \\ e-mail: $\{$ levorato,badia,zorzi\}@ dei.unipd.it.
}

\begin{abstract}
This paper addresses feedback design issues in ad hoc networks with simultaneous access of multiple users, when type II HARQ exploits a constrained capacity reverse channel. In such networks, communications performance is limited by interference and nodes operate in a distributed fashion; thus, efficient resource allocation relies on proper feedback signaling, which on the other hand can use only a limited amount of network resources. For this reason, we investigate how to determine a finite set of feedback messages, which accounts for the underlying type II HARQ error control and jointly regulates power allocation and medium access in an optimal manner.

Index Terms-Feedback channel, ad hoc networks, hybrid automatic repeat request, power control.
\end{abstract}

\section{INTRODUCTION}

The recent advancements in Multi-User Detection (MUD) are driving significant research efforts in the design of networks with simultaneous medium access by the users. In this scenario, proper control of the network load is of paramount importance to preserve the overall performance. The exploitation of feedback from destinations can be an effective solution for distributed systems, where users independently make decisions based on their knowledge of the surrounding conditions, both to improve the resource management [1] and to control the access to the network [2]. However, this message exchange has an impact on the system capacity [3]. To preserve the capacity of the data channel, feedback channels can only convey limited information. In particular, a metric to be sent back to the transmitter can be quantized in order to represent it with a finite number of messages, possibly just a few bits. Examples in this sense can be found in the literature for time- [4] and space-division [5] multiple access networks.

The scenario considered in this paper is a power controlled ad hoc network with type II hybrid automatic retransmission request (HARQ) error control. Every source node encodes the data packet and, after a handshake phase, sends equally-sized codeword fragments to the intended destination. During the handshake and after the transmission of each fragment, the destination sends out a feedback message to report decoding success or failure and to allow power control at the source. Since in type II HARQ the destination combines all the received fragments associated with the same data packet, the knowledge required at the source does not only include current channel conditions, but also the channel conditions experienced by previous transmissions. In particular, the destination reports in the feedback a quantized version of the transmission power required to match a signal-to-noise-plus-interference-ratio (SINR) threshold that depends on the value of the SINR perceived during previous transmissions.

We seek a proper quantization scheme to maximize the throughput/power efficiency of the network. When looking at the whole network, it is key to minimize the power used in each link, and possibly to avoid transmissions which are likely to fail. A maximum power constraint only allows the activation of links that have sufficiently good channel conditions, in terms of both fading and interference. This limits the network load, since when the interference in the network is already high, it is unlikely that further communications can start. Thus, the power limit works as a form of access control. Ongoing attempts can be interrupted as well, but this is less likely to happen since they have lower power requirements, due to already received packets.

Tuning power thresholds to interference and fading statistics can be used to reduce the overall interference. In this computation, the presence of type II HARQ, which affects the transmission process, and thus the interference statistics, must be taken into account. In the end, we show that our power quantization scheme is able to effectively manage power and access control with extremely low signaling overhead.

The rest of this paper is organized as follows. Section II gives an overview of the system under study. Section III develops the analysis. In Section IV we report some numerical results and in Section V we conclude.

\section{System Model}

We consider an ad hoc network, where nodes have to deliver fixed-sized data packets (DPs) to randomly chosen destinations. Nodes try to resolve DP delivery with a type II HARQ error control protocol with power control, so DPs are encoded with a low-rate code and the obtained codeword is split into $F$ HARQ packets (HPs). 


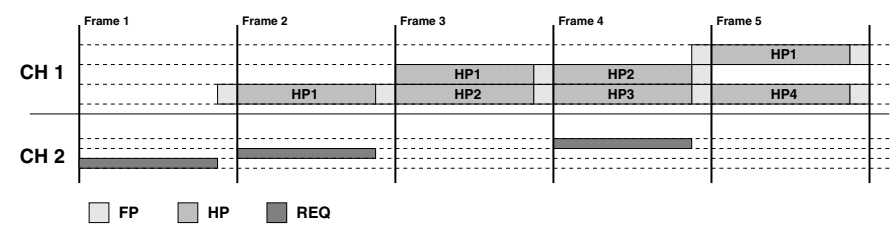

Figure 1. Protocol operations and structure of the channels.

Fig. 1 visually represents the transmission scheme. We assume that time is divided into frames, each subdivided into a data slot and a (shorter) feedback slot. When a node has a packet to deliver, it sends out a request packet (REQ) and the intended destination responds with a feedback packet (FP) during the feedback slot. Then, the source sends the first HP in the data slot after the feedback slot. The HP transmission is followed by a further FP, where the destination reports whether or not the DP was successfully recovered. If it was not, another HP is transmitted, and the data/feedback exchange continues until the DP is acknowledged or the number of HPs sent is equal to $F$. If failure occurs for $F$ HP transmissions a further attempt is scheduled after a random backoff interval. After unsuccessful $D$ attempts, the packet is discarded.

We assume that REQs are sent over a dedicated channel, while HPs and FPs are sent over the same channel, where we allow simultaneous multiple communications. As for transmitter/receiver design, we consider Direct Sequence (DS) Code-division Multiple Access (CDMA) with random sequences, spreading gain $G$ and Matched Filter (MF) receiver. For the sake of simplicity, we assume that FP and REQ are always correctly decoded. This is reasonable, since these short packets are sent with a low information transmission rate. Also, observe that errors occurring in these packets only affect the scheme in the sense of increasing the error rate in the DP exchange. Thus, we assume that errors only occur in the DP exchange and FP and REQ are error-free.

The destination includes in each FP a quantized index to require a certain transmission power, determined by the status of the received HPs and the channel conditions. Note that interfering transmissions may start and end during a communication and the fading coefficients associated with the source/destination link and the various interferer/destination link are time-varying.

The information contained in the FP sent at time $t$ is denoted as $Q_{t}$ and can take value over a finite set with $N+2$ values, which are inferred from an $N$-level quantization of the power values represented by a vector $\boldsymbol{\alpha}=\left(\alpha_{j}\right)$, where $j=1,2, \ldots, N$. For consistency, we also assume $\alpha_{0}=0$. The value of $\alpha_{j}$ is increasing in $j$ and $\alpha_{N}$ (the highest allocable power) is less than or equal to the maximum physical power $P_{\max }$ available at the nodes. This allows to include admission control, i.e., communications requiring very high power, between $\alpha_{N}$ and $P_{\max }$, are discarded. The possible $N+2$ values of $Q_{t}$ include $Q_{t}=1,2, \ldots, N$ to mean that the required power falls in the interval $\left.] \alpha_{Q_{t}-1}, \alpha_{Q_{t}}\right]$, plus two additional values, $Q_{t}=0$ to acknowledge that the DP was successfully extracted, and $Q_{t}=N+1$ to indicate that, due to excessive power requirements the packet has to be discarded.

We assume block flat fading with Rayleigh distribution and independent fading coefficients associated with the various frames. Moreover, we assume a symmetric network, i.e., all links have the same received power statistics, and a Poisson packet generation process with overall birth rate of $\beta \mathrm{pkt} / \mathrm{slot}$. We define the SINR normalized to the transmission power as the quantity

$$
s_{t}=\frac{c_{t}}{\sigma^{2}+\omega_{t}}
$$

where $c_{t}$ is the channel gain coefficient (including pathloss and fading) between the source and the destination, $\sigma^{2}$ is the noise power and $\omega_{t}$ is a random variable taking interference into account. Subscript $t$ refers to the transmission of the $t$ th HP of a communication that, without loss of generality, is also assumed to be the time-index of the frame. The value $t=0$ is associated with the frame preceding the first HP. We further define the vectors, $\mathbf{c}_{t}=\left[c_{0}, \ldots, c_{t}\right], \boldsymbol{\omega}_{t}=\left[\omega_{0}, \ldots, \omega_{t}\right]$ and $\mathbf{s}_{t}=\left[s_{0}, \ldots, s_{t}\right]$. We denote the used power at time $t$ as $P_{t}$, so that the receiver's SINR at time $t$ is $r_{t}=P_{t} s_{t}$. We also write $\mathbf{r}_{t}=\left[r_{0}, \ldots, r_{t}\right]$.

For the coding performance, we assume a threshold model. In particular, we consider good codes where the DP decoding error probability asymptotically vanishes as the codeword length increases if the channel parameters fall in the so-called reliable region [6]. Since we rely on SINR values to define channel evolution, in the type II HARQ case, upon the reception of the $t$ th HP the reliable region $\mathcal{S}_{t}$ is a $t$-dimensional region where the DP decoding error probability is zero if $\mathbf{s}_{t} \in \mathcal{S}_{t}$ and one otherwise. We define the boundary function

$$
\vartheta\left(\mathbf{r}_{t}\right)= \begin{cases}\inf \left\{r_{t+1}:\left[\mathbf{r}_{t} r_{t+1}\right] \in \mathcal{S}_{t+1}\right\} & \text { if } \mathbf{r}_{t} \notin \mathcal{S}_{t} \\ 0 & \text { otherwise }\end{cases}
$$

that returns the lowest SINR value required for correct decoding, given the previous SINR values. Note that the DP is successfully decoded upon the first transmission if $r_{1}$ is greater than a constant threshold $\vartheta_{1}$.

Since the SINR vector $\mathbf{s}_{t}$ is a deterministic function of the channel gains vector $\mathbf{c}_{t}$ and the interference vector $\boldsymbol{\omega}_{t}{ }^{1}$ we map the region $\mathcal{S}_{t}$ to the region $\mathcal{R}_{t}$ of vector pairs $\left(\boldsymbol{\omega}_{t}, \mathbf{c}_{t}\right)$, i.e.,

$$
\mathcal{R}_{t}= \begin{cases}\left\{\left(\boldsymbol{\omega}_{t}, \mathbf{c}_{t}\right): \mathbf{r}_{t} \in \mathcal{S}_{t}\right\} & 1<t \leq F \\ \emptyset & t=1 .\end{cases}
$$

\footnotetext{
${ }^{1}$ The SINR at frame $t$ is a function of $\omega_{t}, c_{t}$, and the transmission power, that is a function of the previously perceived SINR values.
} 
We define the power control function $\Phi_{t}$, that maps the values of $\omega_{t}$ and $c_{t}$ to the power required for correct decoding at the next HP transmission, also including an SINR margin $\epsilon$ to account for channel and interference variations. After the handshake, this power value is

$$
\Phi_{0}\left(\omega_{0}, c_{0}\right)=\frac{\sigma^{2}+\omega_{0}}{c_{0}}\left(\vartheta_{1}+\epsilon\right),
$$

whereas for a frame with index $t>0$,

$$
\Phi_{t}\left(\boldsymbol{\omega}_{t}, \mathbf{c}_{t}\right)=\frac{\sigma^{2}+\omega_{t}}{c_{t}}\left(\vartheta\left(\mathbf{r}_{t}\right)+\epsilon\right) .
$$

Thus, with the $t$-th FP, the destination sends back to the source a quantized feedback index $Q_{t}$. If the destination successfully decoded the DP, i.e., $\left(\boldsymbol{\omega}_{t}, \mathbf{c}_{t}\right) \in \mathcal{R}_{t}$, an acknowledgement is sent back, which is denoted with $Q_{t}=0$. If the required power is higher than $\alpha_{N}$, the destination includes in the feedback message the value $Q_{t}=N+1$, which tells the source to refrain from additional HP transmissions. A further attempt can be rescheduled if the maximum number of attempts has not been reached yet. Otherwise, the quantization index denotes the power to use in the transmission of the $(t+1)$ th HP, which will be $P_{t+1}=\alpha_{Q_{t}}$. To sum up,

$$
Q_{t}= \begin{cases}0 & \text { if resolved DP } \\ N+1 & \text { if aborted DP } \\ \arg \min _{k}\left\{\alpha_{k} \geq \Phi_{t}\left(\boldsymbol{\omega}_{t}, \mathbf{c}_{t}\right)\right\} & \text { otherwise }\end{cases}
$$

We also define the region

$$
\mathcal{J}_{t}=\left\{\left(\boldsymbol{\omega}_{t}, \mathbf{c}_{t}\right): \Phi_{t}\left(\boldsymbol{\omega}_{t}, \mathbf{c}_{t}\right)>\alpha_{N}\right\},
$$

describing the region where the transmission is interrupted due to poor channel conditions. Similarly, $\mathcal{U}_{t}=$ $\mathcal{R}_{t} \cup \mathcal{J}_{t}$ denotes the general transmission termination region (due to either poor channel or correct reception).

\section{Analytical Framework}

The radio resource allocation has to deal with interference and fading statistics. Note that in general interference terms $\omega_{i}$ are correlated due to retransmissions and power control. In fact, the presence of an interferer influences the outcome of both communications already ongoing and those in handshake phase. Thus, for instance, to track the first transmission of a HP packet, we must take into account that the presence of interferers in the preceding slots influences the transmission power of such a packet. Since the index of the first idle slot is also a random variable, that furthermore typically has a large value with high probability under heavy aggregated traffic conditions, the evaluation of the exact statistics of $\omega_{0}$ appears to be a formidable task. Also, the interactions among interfering communications are very complex in general. For these reasons, we set up an approximated recursive analytical algorithm, in order to preserve accuracy while guaranteeing affordable complexity. By our approach, the statistics of interfering communications (power, length, duration) are approximated with their averages, computed recursively based on those derived previously. In particular, at each iteration we derive the statistics of a single communication, from which we compute the average interference behavior for the next iteration.

We define the conditional decoding failure and general attempt interruption probabilities:

$$
\begin{aligned}
\rho_{t} & =\mathcal{P}\left\{\left(\boldsymbol{\omega}_{t}, \mathbf{c}_{t}\right) \notin \mathcal{R}_{t} \mid\left(\boldsymbol{\omega}_{t-1}, \mathbf{c}_{t-1}\right) \notin \mathcal{U}_{t-1}\right\} \\
\mu_{t} & =\mathcal{P}\left\{\left(\boldsymbol{\omega}_{t}, \mathbf{c}_{t}\right) \in \mathcal{U}_{t} \mid\left(\boldsymbol{\omega}_{t-1}, \mathbf{c}_{t-1}\right) \notin \mathcal{U}_{t-1}\right\}
\end{aligned}
$$

The average transmission power at the $t$-th transmission, given that the $t$-th transmission occurs, is $\bar{P}_{t}=\sum_{k=1}^{N} \alpha_{k} A_{t}(k)$, where $A_{t}(k)$ is defined in (9) and represents the probability that at the $t$-th transmission the source uses power $\alpha_{k}, 0<k \leq N$. The notation $\mathbb{E}\left[\mathcal{P}\left\{e v_{1} \mid e v_{0}\right\}\right]$ denotes the expected value of the probability of the event $e v_{1}$ given a conditioning event $e v_{0}$, where the expectation is taken over all possible interference values.

To keep the problem tractable we approximate the power transmitted by a given interferer in a given slot with its average $\bar{P}$. In particular, $\bar{P}$ is the average power transmitted by an interfering node in a randomly selected slot (we do not keep track of the index of the HP transmitted by the interferer in the current slot). Define an $F$-state Markov chain with states $1, \ldots, F$, where the only transitions from each state $j$ are to state 1 with probability $\mu_{j}$ or to state $(j \bmod F)+1$ with probability $1-\mu_{j}$. The steady-state probability $x_{j}$ of the Markov chain being in state $j$ can be derived as [7]

$$
x_{j}=\frac{\prod_{h=1}^{j}\left(1-\mu_{h}\right)}{1+\sum_{i=1}^{F} \prod_{h=1}^{i}\left(1-\mu_{h}\right)} .
$$

$\bar{P}$ can be obtained by weighing $\bar{P}_{t}$ with the probabilities of the interferer being at the $t$ th transmission, which in turn are taken equal to the steady-state probabilities $x_{t}$ of the Markov chain.

$$
\begin{aligned}
A_{t}(k) & =\mathcal{P}\left\{\alpha_{k-1}<\Phi_{t-1}\left(\boldsymbol{\omega}_{t-1}, \mathbf{c}_{t-1}\right) \leq \alpha_{k}\right\}=\mathbb{E}\left[\mathcal{P}\left\{P_{t}=\alpha_{k} \mid\left(\mathbf{c}_{t-1}, \boldsymbol{\omega}_{t-1}\right) \notin \mathcal{U}_{t-1}\right\}\right] \\
& =\mathbb{E}\left[\mathcal{P}\left\{\alpha_{k}<\frac{\sigma^{2}+\omega_{t}}{c_{t}}\left(\vartheta\left(\mathbf{r}_{t-1}\right)+\epsilon\right) \leq \alpha_{k+1} \mid\left(\mathbf{c}_{t-1}, \boldsymbol{\omega}_{t-1}\right) \notin \mathcal{U}_{t-1}\right\}\right]
\end{aligned}
$$


Under the symmetric network hypothesis, we assume that any coefficient $C$ associated with interfering transmissions has the same probability density function and that it is Rayleigh, i.e.,

$$
f_{C}(c)=\frac{1}{\ell} e^{-\frac{c}{\ell}}, \quad c \geq 0
$$

where $\ell$ is the average path loss coefficient. Thus, $\omega_{i}$ are sums of $Z_{i}$ i.i.d. contributions, where $Z_{i}$ is the number of interferers in slot $i$. The interfering power is the sum of $Z_{i}$ Rayleigh terms, and therefore has Gamma statistics with parameters $Z_{i}$ and $2 \nu / G$, where $\nu=\bar{P} \ell$, and probability density function $f_{\omega_{i} \mid z_{i}}(\omega)$.

We now derive the statistics of the number of interfering transmissions $Z_{i}$ at the various frames of the communication. Under the previously stated assumptions, the system performance only depends on the $Z_{i}$ terms. We denote with $\mathbf{Z}$ the $F$-sized vector containing the values of $Z_{i}$, and we derive the fundamental radio resource allocation metrics given a specific realization $\mathbf{Z}=\mathbf{z}$.

We define the cumulative distribution of $s_{i}$ values

$$
\begin{aligned}
\mathcal{F}_{s_{i}}^{z_{i}}\left(s^{*}\right) & =\mathcal{P}\left\{s_{i} \leq s^{*} \mid Z_{i}=z_{i}\right\} \\
& =\int_{0}^{+\infty} f_{\omega_{i} \mid z_{i}}(\omega) \int_{0}^{\left(s^{*}\left(\sigma^{2}+\omega\right)\right)} f_{C}(c) \mathrm{d} c \mathrm{~d} \omega, \\
& =1-e^{-\frac{s^{*} \sigma^{2}}{\ell}}\left(\frac{2 s^{*}}{\ell}+\frac{1}{\nu}\right)^{-z_{i}} \nu^{-z_{i}} .
\end{aligned}
$$

The probability that the attempt is interrupted before the first $\mathrm{HP}$ transmission is $\mu_{0}=1-\mathcal{F}_{s_{0}}^{z_{0}}\left(\left(\vartheta_{1}+\epsilon\right) / \alpha_{N}\right)$. Given that this did not occur, the distribution of the power used in the next frame is

$$
A_{1}(k)=\left(\mathcal{F}_{s_{0}}^{z_{0}}\left(\frac{\vartheta_{1}+\epsilon}{\alpha_{k-1}}\right)-\mathcal{F}_{s_{0}}^{z_{0}}\left(\frac{\vartheta_{1}+\epsilon}{\alpha_{k}}\right)\right) /\left(1-\mu_{0}\right),
$$

and the failure probability is

$$
\begin{aligned}
\rho_{1} & =\frac{\mathcal{P}\left\{r_{1} \leq \vartheta_{1}, \frac{\vartheta_{1}+\epsilon}{\alpha_{N}}>s_{0}\right\}}{1-\mu_{0}} \\
& =\sum_{k=1}^{N} A_{1}(k) \mathcal{P}\left\{r_{1} \leq \vartheta_{1} \mid A_{1}=\alpha_{k}\right\} \\
& =\sum_{k=1}^{N} A_{1}(k) \mathcal{F}_{s_{1}}^{z_{1}}\left(\frac{\vartheta_{1}}{\alpha_{k}}\right) .
\end{aligned}
$$

The probability that the second HP transmission takes place is as in (15). Eqs. (16) and (17) give the expressions for $A_{2}(k)$ and $\rho_{2}$, respectively. These lead to integral expressions of $\mathcal{F}_{s_{i}}^{z_{i}}\left(s^{*}\right)$, whose integration region depends on $\vartheta$. Analogous formulations are available for further transmissions. The target of our quantization is to maximize the average throughput

$$
\eta=R \beta \sum_{t=1}^{F} \frac{1-\rho_{t}}{t} \prod_{h=0}^{t-1}\left(1-\mu_{h}\right) \quad[\mathrm{bit} / \mathrm{s} / \mathrm{Hz}],
$$

where $R$ is the transmission rate. We assume $R$ to be equal for all terminals, even though our scheme can be easily applied even without this assumption, which is just introduced to better present the numerical results, where $R$ is taken as the independent variable. The average throughput per unit of power is

$$
\psi=R \beta \sum_{t=1}^{F} \frac{1-\rho_{t}}{t \bar{P}_{t}} \prod_{h=0}^{t-1}\left(1-\mu_{h}\right) .
$$

\section{A. Number of Interfering Transmissions}

We define $\Psi(u)$ as the probability that an attempt has duration less than or equal to $u$ frames.

$$
\Psi(u)=\sum_{t=1}^{u}\left(\mu_{t} \prod_{h=1}^{t-1}\left(1-\mu_{h}\right)\right), \text { if } 1 \leq u<F,
$$

and $\Psi(u)=1$ if $u=F$. We also define the average duration $t_{a v}=1+\sum_{u=1}^{F}(1-\Psi(u))$ and the average number of active communications $\lambda=\left(1-\mu_{0}\right) \beta$.

We denote as $Y_{i}$ the number of interferers active in frame 0 that are still active in frame $i$. Thus, $Y_{0}$ is the number of ongoing transmissions during the handshake, and its distribution is [7]

$$
\pi_{y_{0}}=\mathcal{P}\left\{Y_{0}=y_{0}\right\}=\frac{\left(\lambda t_{a v}\right)^{y_{0}} e^{-\lambda t_{a v}}}{y_{0} !} \text {, for } y_{0}=0,1, \ldots
$$

The probability that one ongoing transmission in frame 0 is still active after $n$ frames is [7]

$$
\tau(n)=1-\frac{1}{t_{a v}} \sum_{u=1}^{n}(1-\Psi(u)) .
$$

$$
\begin{aligned}
\mu_{1}= & 1-\rho_{1}+\sum_{k_{1}=1}^{N} A_{1}\left(k_{1}\right) \mathcal{P}\left\{\alpha_{k_{1}} s_{1}<\vartheta_{1}\right\} \mathcal{P}\left\{\frac{\vartheta\left(\alpha_{k_{1}} s_{1}\right)+\epsilon}{s_{1}}>\alpha_{N} \mid \alpha_{k_{1}} s_{1}<\vartheta_{1}, \frac{\vartheta_{1}+\epsilon}{\alpha_{N}}<s_{0}\right\} \\
A_{2}(k)= & \sum_{k_{1}=1}^{N} \frac{A_{1}\left(k_{1}\right)}{1-\mu_{1}} \mathcal{P}\left\{\alpha_{k-1} \leq \frac{\vartheta\left(\alpha_{k_{1}} s_{1}\right)+\epsilon}{s_{1}}<\alpha_{k}, \frac{\vartheta\left(\alpha_{k_{1}} s_{1}\right)+\epsilon}{s_{1}}<\alpha_{N}, s_{1} \leq \frac{\vartheta_{1}}{\alpha_{k_{1}}}\right\} \\
\rho_{2}= & \sum_{k_{2}=1}^{N} \sum_{k_{2}=1}^{N} A_{1}\left(k_{1}\right) A_{2}\left(k_{2}\right) \mathcal{P}\left\{\alpha_{k_{2}} s_{2} \leq \vartheta\left(\alpha_{k_{1}} s_{1}\right), \alpha_{k_{2}-1} \leq \frac{\vartheta\left(\alpha_{k_{1}} s_{1}\right)+\epsilon}{s_{1}}<\alpha_{k_{2}}, s_{1} \leq \frac{\vartheta_{1}}{\alpha_{k_{1}}}, \frac{\vartheta_{1}+\epsilon}{\alpha_{N}}<s_{0}\right\}
\end{aligned}
$$


Thus, we define the probability

$$
\begin{aligned}
& \mathcal{P}\left\{Y_{i}=y_{i} \mid Y_{0}=y_{0}\right\}= \\
& \begin{cases}\left(\begin{array}{l}
y_{0} \\
y_{i}
\end{array}\right) \tau(i)^{y_{i}}(1-\tau(i))^{y_{0}-y_{i}} & \text { if } y_{i} \leq y_{0} \\
0 & \text { if } y_{i}>y_{0} .\end{cases}
\end{aligned}
$$

Note that we have $Y_{F}=0$, as the number of attempts can not be larger than $F$.

We define $W_{i}$ as the number of interfering transmissions that start at frames with index greater than or equal to 1 and are still alive at frame $i$.

$$
\mathcal{P}\left\{W_{i}=w_{i}\right\}=\frac{e^{-\zeta_{i}} \zeta_{i}^{w_{i}}}{w_{i} !}, \text { for } w_{0}=0,1, \ldots
$$

where

$$
\zeta_{i}= \begin{cases}\lambda+\lambda \sum_{j=1}^{i-1}(1-\Psi(j)) & \text { if } i>1 \\ \lambda, & \text { if } i=1\end{cases}
$$

The distribution of the total number of active interferers in the $i$-th frame, given their number in frame 0 (note that $\left.z_{0}=y_{0}\right)$ is $\pi_{z_{i} \mid z_{0}}$ as in (26). We are then able to associate to each vector $\mathbf{z}$ the probability $\mathcal{P}\{\mathbf{Z}=\mathbf{z}\}=$ $\pi_{z_{0}} \prod_{i=1}^{F} \pi_{z_{i} \mid z_{0}}$.

\section{B. Recursive Analysis and Quantization Optimization}

The analysis presented above enables the derivation of overall network metrics given the vector of the number of active interferers $\mathbf{z}$ and also the computation of the probability of $\mathbf{z}$. To both evaluate the efficiency of the resource allocation and optimize the quantization vector we set up a recursive algorithm. Recursion is needed since the performance of a node depends on the interference statistics, which is in turn determined by the statistics of each single node.

We start assessing the metrics in the absence of interference and then we evaluate the distribution of the number of interfering nodes. Using this as the initial distribution, we are able to average the performance on the number of interfering nodes and to optimize the threshold vector with a constrained search algorithm. Given the obtained thresholds, we can recompute the performance and interference statistics, optimize the thresholds again, and so on.

Due to the retransmission policy, the birth process rate is increased. In particular, the total transmission birth rate is $\beta^{\prime}=\beta \Delta$, where $\Delta$ is the average number of attempts where at least one HP is transmitted. We define the events $e v_{(A)}, e v_{(F)}$ and $e v_{(S)}$ as an aborted attempt before the first HP is sent, a failed or interrupted attempt due to excessive required transmission power, and a successful attempt, respectively. The probabilities of these events are

$$
\begin{aligned}
& \mathcal{P}\left\{e v_{(A)}\right\}=\mu_{0}, \\
& \mathcal{P}\left\{e v_{(F)}\right\}=1-\mu_{0}-\sum_{t=1}^{F}\left(1-\rho_{t}\right) \prod_{h=0}^{t-1}\left(1-\mu_{h}\right), \\
& \mathcal{P}\left\{e v_{(S)}\right\}=\sum_{t=1}^{F}\left(1-\rho_{t}\right) \prod_{h=0}^{t-1}\left(1-\mu_{h}\right) .
\end{aligned}
$$

We define $\mathcal{E}$ as the set of all the possible patterns of events $e v_{(A)}, e v_{(F)}$ and $e v_{(S)}$ associated with a packet. For a specific packet, we denote with $\Xi_{j} \in \mathcal{E}$ the event occurring at the $j$ th transmission, and we collect the $\Xi_{j}$ values into a vector $\Xi$. Note that the event $e v_{(S)}$ terminates the transmission of the packet. Thus, either $\boldsymbol{\Xi}$ is of length $D$ or it ends with a success event and it has length $L(\boldsymbol{\Xi}) \leq D$, where $\Xi_{L(\boldsymbol{\Xi})}=e v_{(S)}$. The probability of a given instance $\boldsymbol{\xi}$ of $\boldsymbol{\Xi}$ is $\mathcal{P}\{\boldsymbol{\xi}\}=\prod_{j=1}^{L(\boldsymbol{\xi})} \mathcal{P}\left\{\xi_{j}\right\}$. Calling $\mathcal{X}$ the set of all possible $\xi \mathrm{s}$, and with $|\cdot|$ the set cardinality operator, the average number of attempts with at least one transmitted HP is

$$
\Delta=\sum_{\boldsymbol{\xi} \in \mathcal{X}} \mathcal{P}\{\boldsymbol{\xi}\} \sum_{j=1}^{L(\boldsymbol{\xi})}\left|\left\{\xi_{j}\right\} \cap\left\{e v_{(S)}, e v_{(F)}\right\}\right|,
$$

\section{NUMERICAL RESULTS}

In this section, we compare our proposed feedback design procedure with a uniform power quantization, i.e., $\alpha_{j}=j P_{\max } / N$. Note that no admission control is performed in this case, since $\alpha_{N}=P_{\max }$.

The HARQ thresholds are defined by assuming the codes are capacity-achieving. Thus, the required SINR to correctly decode the DP after having received $t \mathrm{HP}$ is

$$
\vartheta\left(\mathbf{r}_{t}\right)=\inf \left\{r_{t+1}: \sum_{j=1}^{t+1} \log _{2}\left(1+r_{j}\right) \geq R\right\}
$$

This capacity criterion relates to the well-known Shannon bound, and it is well approached in practice by socalled good codes [6].

In the following, we present results for a system with two thresholds and two maximum allowed HP transmissions per attempt, i.e., $N=2$ and $F=2$. We assume that the received power decays according to a

$$
\pi_{z_{i} \mid z_{0}}=\mathcal{P}\left\{Z_{i}=z_{i} \mid Z_{0}=z_{0}\right\}=\sum_{u=0}^{\min \left(z_{i}, z_{0}\right)} \frac{z_{0} !}{u !\left(z_{0}-u\right) !} \tau(i)^{u}(1-\tau(i))^{z_{0}-u} \frac{e^{-\lambda i p}(\lambda i p)^{z_{i}-u}}{\left(z_{i}-u\right) !}
$$




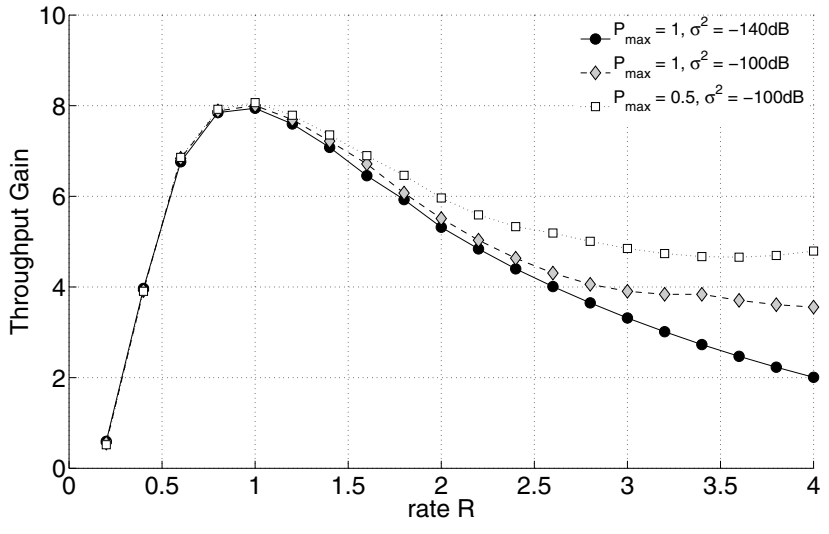

Figure 2. Throughput vs. transmission rate.

path loss which is as a power of the distance with exponent equal to 4 . The intensity of the arrival process is $\beta=3 \mathrm{pkt} / \mathrm{slot}$. We show in Figs. 2 and 3 the throughput gain and the throughput per unit of power gain between the optimal and uniformly placed thresholds as a function of the transmission rate $R$.

We compare three different parameter settings: $P_{\max }=$ $1 \mathrm{~W}, \sigma^{2}=-140 \mathrm{dBW} ; P_{\max }=1 \mathrm{~W}, \sigma^{2}=-100 \mathrm{dBW}$; $P_{\max }=0.5 \mathrm{~W}, \sigma^{2}=-100 \mathrm{dBW}$. The first setting has almost negligible noise power and the system performance is interference constrained. In the two last settings, differing only in the maximum power, the system performance is both interference and noise constrained.

In all settings the optimal quantization has a throughput gain which is more evident for intermediate values of the transmission rate $R$. In fact, for low values of $R$ the system is able to manage interference and noise due to the low SINR requirements, whereas for high values of $R$ even the optimized systems can not achieve the high SINR required and aborts attempts with high probability. For intermediate values of $R$, the feedback optimization significantly increases the SINR at the destination.

Results not presented here for lack of space show that the power thresholds selected in the optimized system have a different behavior for the two values of $\sigma^{2}$. In the noise and interference constrained setting the average transmitted power generally increases as $R$ is increased, whereas in the interference constrained setting the average transmitted power decreases as $R$ is increased. In the former case the system has generally to increase the transmitted power in order to match the SINR requirements due to the large noise power. This effect is more noticeable in the case with $P_{\max }=0.5$ $\mathrm{W}$, since the uniform case allocates a threshold in 0.25 $\mathrm{W}$, that generates transmissions with too low power for this noisy channel. In the latter case, the system is able to reduce the transmitted power for transmissions with already received HPs or enjoying good channel conditions. Thus, the system generally sets a threshold at low power to save power for transmissions with low

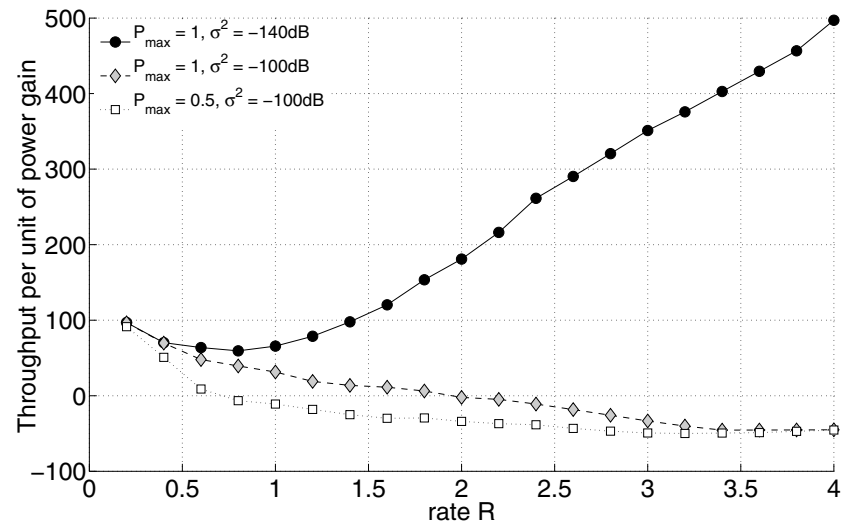

Figure 3. Throughput per unit of power vs. transmission rate.

SINR requirements and an intermediate-power threshold to guarantee link activation.

Fig. 3 shows that while in the interference constrained setting the system spends also a lower amount of power, in the noise and interference constrained case the system achieves a better performance in terms of throughput at the price of an increased cost in terms of power.

\section{Conclusions}

In this paper, we investigated the impact of the feedback control design on power control in ad hoc networks with MUD and type-II HARQ. We focused on a receiver-driven power control where feedback packets are exchanged containing an index of the power required to correctly achieve the incremental redundancy needed for correct data reception. We showed that a proper selection of quantization intervals for the power values to be fed back to the transmitter's side can achieve significant performance improvement, especially in terms of increased throughput per unit power.

\section{REFERENCES}

[1] T. Yoo, N. Jindal, and A. Goldsmith, "Multi-antenna dowlink channels with limited feedback and user selection," IEEE J. Sel. Areas Commun., vol. 25, no. 7, pp. 1478-1491, Sep. 2007.

[2] V. Hassel, D. Gesbert, M. S. Alouini, and G. E. Oien, "A threshold-based channel state feedback algorithm for modern cellular systems," IEEE Trans. Wireless Commun., vol. 6, no. 7, pp. 2422-2426, Jul. 2007.

[3] P. Xia, S. Zhou, and G. B. Giannakis, "Multiantenna adaptive modulation with beamforming based on bandwidth-constrained feedback," IEEE Trans. Commun., vol. 53, no. 3, pp. 526-536, Mar. 2005.

[4] A. P. T. Lau and F. R. Kschischang, "Feedback quantization strategies for multiuser diversity systems," IEEE Trans. Inf. Theory, vol. 53, no. 4, pp. 1386-1400, Apr. 2007.

[5] K. Huang, R. W. Heath, and J. G. Andrews, "Space division multiple access with a sum feedback rate constraint," IEEE Trans. Signal Process., vol. 55, no. 7, pp. 3879-3891, Jul. 2007.

[6] R. Liu, P. Spasojevic, and E. Soljanin, "Reliable channel regions for good binary codes transmitted over parallel channels," IEEE Trans. Inf. Theory, vol. 52, no. 4, pp. 1405-1424, Apr. 2006.

[7] S. Karlin and H. M. Taylor, An introduction to stochastic modeling. San Diego: Academic Press, 1998. 